\title{
Magnetic field topology during July 14-16 2000 (Bastille Day) solar CME event
}

\author{
L. Rastätter, M. Hesse, and M. Kuznetsova \\ NASA/GSFC, Greenbelt, Maryland, USA
}

\section{T. I. Gombosi and D. L. DeZeeuw}

Department of Engineering, University of Michigan, Ann Arbor MI, USA

Received 19 September 2001; revised 13 March 2002; accepted 13 March 2002; published 14 August 2002.

[1] The July 14-16, 2000 (Bastille Day) event was marked by an extraordinary level of geomagnetic activity following the impact of a large magnetic cloud onto the Earth's magnetosphere at 14:38 on July 15, 2000. The strong increase of the solar wind speed, and density and the large amplitude and variability of $B_{y}$ and $B_{z}$ gives rise to dramatic topological changes of unexpected magnitude in the magnetic field of the near-Earth magnetosphere. This event is one of the first real events comprehensively modeled with the adaptive grid MHD simulation scheme BATS-R-US. Magnetic field line integrations were performed with the model output to obtain the three-dimensional magnetic topology and the magnetic mapping between the northern and the southern hemisphere of the Earth. INDEX TERMS: 2753 Magnetospheric Physics: Numerical modeling; 2788 Magnetospheric Physics: Storms and substorms; 2784 Magnetospheric Physics: Solar wind/magnetosphere interactions; 2744 Magnetospheric Physics: Magnetotail

\section{Introduction}

[2] In this paper we describe the changes in magnetic topology during the time after the first impact of the coronal mass ejection (CME) event at about 14:38 UT on July 15, 2000. The simulation covers the time period from 14:00 UT to about 21:20 UT, but in this paper we focus on the time interval from 14:28 UT to 15:16 UT (Figure 1, vertical solid lines). The CME structure passing the GEOTAIL satellite around 14:35 UT (dashed lines) is characterized by $B_{z}$ changing from a steady value of $-3 \mathrm{nT}$ to $+15 \mathrm{nT}$ between 14:38 UT and 14:45 UT. $B_{y}$ rises sharply from $\sim 2 \mathrm{nT}$ to $+20-+25 \mathrm{nT}$ around 14:35 UT. The solar wind speed $V_{x}$ jumps from $-600 \mathrm{~km} / \mathrm{s}$ (in GSM) to $-900 \mathrm{~km} / \mathrm{s}$ and the plasma density $N$ rises from a quiet-time level of $5 \mathrm{~cm}^{-3}$ to over $20 \mathrm{~cm}^{-3}$ during the 30 minutes of the passage of this first CME structure. The solar wind ram pressure $\left(\frac{\rho}{2} V_{x}^{2}\right)$ increases from $\sim 20 \mathrm{nPa}$ to $200 \mathrm{nPa}$ during this time and falls back to $30 \mathrm{nPa}$ by 15:24 UT. At the trailing edge of the disturbance $B_{y}$ reverses to $\sim-30 \mathrm{nT}$ between 15:06 UT to 15:18 UT and the particle density reverts to previous levels of $\sim 2-3 \mathrm{~cm}^{-3}$. The solar wind velocity $V_{x}$, however, remains between $-900 \mathrm{~km} / \mathrm{s}$ and $-1000 \mathrm{~km} / \mathrm{s}$ throughout the rest of the time interval. We will study effects occurring on short time scales while the magnetosphere contracts and expands again and magnetic reconnection changes the magnetic field line mapping on the Earth.

[3] This study focuses on the three-dimensional structure of the near-Earth magnetospheric magnetic field responding to the solar wind. For this purpose we employ the Block Adaptive Tree Solarwind Roe Upwind Scheme (BATS-RUS) [Powell et al., 1999; Gombosi et al., 2002]. Models such as BATS-R-US are tested at the Community Coordinated Modeling Center (CCMC, http://ccmc.gsfc.nasa.gov/) for space weather purposes.

\section{The MHD Model}

[4] BATS-R-US uses a conservative form of the ideal magnetohydrodynamics (MHD) equations in a semi-relativistic approximation to solve for the MHD variables density, pressure, velocity, and magnetic field $(\rho, P, \mathbf{V}, \mathbf{B}$, respectively) The semi-relativistic Boris-correction-type approach [Boris, 1970; Gombosi et al., 2002] employed in BATS-RUS limits the maximum Alfvén speed near the Earth to the artificially reduced speed of light $c(c=6000 \mathrm{~km} / \mathrm{s}$, well above plasma speeds modeled in our run) and thus allows for global-scale modeling at high spatial resolution. For an in-depth discussion on the equations and solution methods see Gombosi et al. [2002] and Powell et al. [1999].

[5] The simulation box size ( $\mathrm{R}_{\mathrm{E}}$, in GSM coordinates) is $-360<X<24,-96<Y<96$, and $-96<Z<96$ with roughly 800,000 grid cells. Block cell size (edge length) ranges from $8 R_{E}$ in the far end of the magnetotail to as low as $1 / 4 \mathrm{R}_{\mathrm{E}}$ in blocks nearest to the Earth (where $|X|$, $|Y|,|Z|<6)$. The bow shock region within $12 \mathrm{R}_{\mathrm{E}}$ and the tail plasma sheet for $X>-24$ are resolved with cells of $1 / 2 \mathrm{R}_{\mathrm{E}}$. The upstream inflow boundary (at $X=24$ ) is updated with GEOTAIL magnetic field [Kokobun et al., 1994] and CPI plasma speed, density and temperature data [Frank et al., 1994] (see Figure 1). GEOTAIL's location was between 23 $\mathrm{R}_{\mathrm{E}}$ and $25 \mathrm{R}_{\mathrm{E}}$ in $X$ and its offset from the Sun-Earth line was small $\left(|Y|,|Z|<4 \mathrm{R}_{\mathrm{E}}\right)$ during the hours simulated. The side boundaries (at $Y= \pm 96$ and $Z= \pm 96$ ) have zero-gradient boundary conditions. Outflow boundary conditions are specified at the far-tail boundary $(X=-360)$.

[6] The inner boundary of the magnetospheric MHD simulation is located at $3 R_{E}$ from the Earth to avoid the extremely high magnetic field and high Alfvén speeds close to the Earth. The MHD portion of the model is coupled to an ionospheric potential solver [Goodman, 1995; Amm, 1996; Goodman, 1996; Sojka et al., 1997] to obtain self-consistent plasma flow conditions at the 


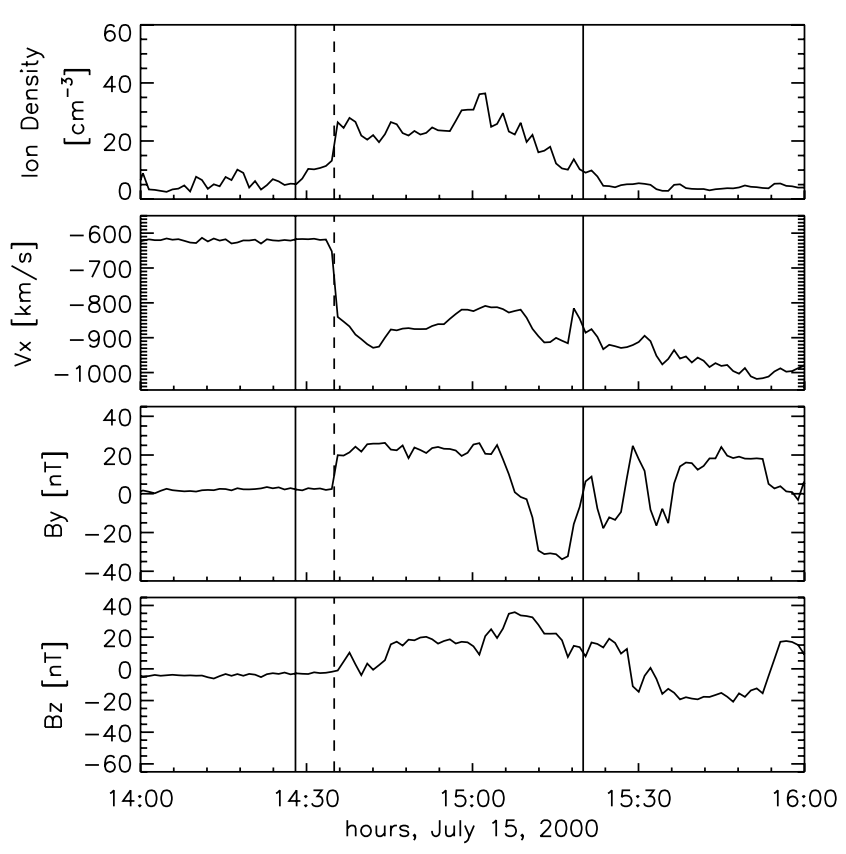

Figure 1. Solar wind input. For the period from 14:00 UT to 16:00 UT, the solar wind parameters used in the simulation, as measured by GEOTAIL, are shown: plasma density $N$, velocity $V_{x}$, and magnetic field components $B_{y}$, $B_{z}$. The vertical bars show the time period that is addressed in this paper (solid lines: 14:28 UT and 15:16 UT), the dashed lines show the arrival time (14:35 UT) of the interplanetary shock at the satellite.

inner boundary of the MHD region. To accomplish this, field-aligned electric currents from near the MHD boundary are mapped to the ionosphere along magnetic dipole model field lines. An ionospheric conductance model based on Rasmussen and Schunk [1987] provides for the electric potential solution using the given field-aligned currents. Electric fields are mapped back to the boundary of the MHD region to provide for plasma convection. Conductances in the model ionosphere range up to $27.9 \mathrm{~S}$ for Hall and $19 \mathrm{~S}$ for Pedersen conductivities in the dayside with a solar microwave parameter F10.7 $=213$ for that day. Nightside backgrounds are $1 \mathrm{~S}$ and $0.5 \mathrm{~S}$, respectively. The ionosphere calculation and mapping are done once every minute of simulated time and the dipole field orientation in the GSM $X-Z$ plane is updated at a similar frequency according to the Earth's rotation. With typical solar wind velocity of $-600--1000 \mathrm{~km} / \mathrm{s}$, solar wind conditions seen at GEOTAIL at $24 \mathrm{R}_{\mathrm{E}}$ pass the Earth about 3-4 minutes later. The code assumes a steady state after a short time-independent setup phase and typically 10-15 minutes of time-dependent simulation with constant solar wind input. Thus dynamic changes of magnetospheric and magnetotail conditions are expected to be realistically modeled as we start the run early enough ( $\sim 20$ minutes) before the initial shock front hits the magnetosphere. The IMF $B_{x}$ is set to zero throughout the simulations as $\nabla \cdot \mathbf{B}=0$ has to be fulfilled at the upstream boundary and $B_{y}$ and $B_{z}$ are assumed to be constant on the box surface at $X=24$.

\section{Results}

[7] The shock arrives at GEOTAIL around 14:35 UT (Figure 1) and reactions in the inner magnetospheric are seen shortly afterward. Figure 2 shows the changing magnetospheric conditions during the time period between 14:28 UT and 15:16 UT in the meridional plane $Y=0$. The two panels show plasma pressure (grayscale) before shock arrival at the magnetopause (14:28 UT, left) and at the end of the time period (15:16 UT, right). The increased ram pressure causes the tail to shrink from the initial quiet state at 14:28 to its turbulent state later at 15:16 UT. The plasma pressure along the magnetopause reaches up to $11.8 \mathrm{nPa}$ at the later time and enhanced pressure in the plasma sheet is clearly visible. The tail flaps violently during this time period as IMF conditions change, especially large and varying perpendicular components $B_{y}$ and $V_{y}$ together with the reversing $B_{z}$ move the plasma sheet away from the $X$ axis as seen in the 15:16 UT panel.

[8] Figure 3 shows the magnetic field lines outside of 3 $\mathrm{R}_{\mathrm{E}}$, the inner boundary of the MHD part of the simulation. Open field lines connecting the Earth polar regions to the IMF (dark shade emanating from the north, black from the south) show the symmetric distribution formed in the steady southward IMF condition up to 14:28 UT (left panel). By 14:52 UT (middle), the amount of open flux has decreased dramatically and the east-west distribution of the IMF connections has become asymmetrical caused by the strong $B_{y}$. The dawn-dusk connectivity is now clearly seen in the projection onto the cross-sectional plane. The north-south direction of the IMF field the open field lines are connected to has reversed with the now positive (northward) IMF $B_{z}$.
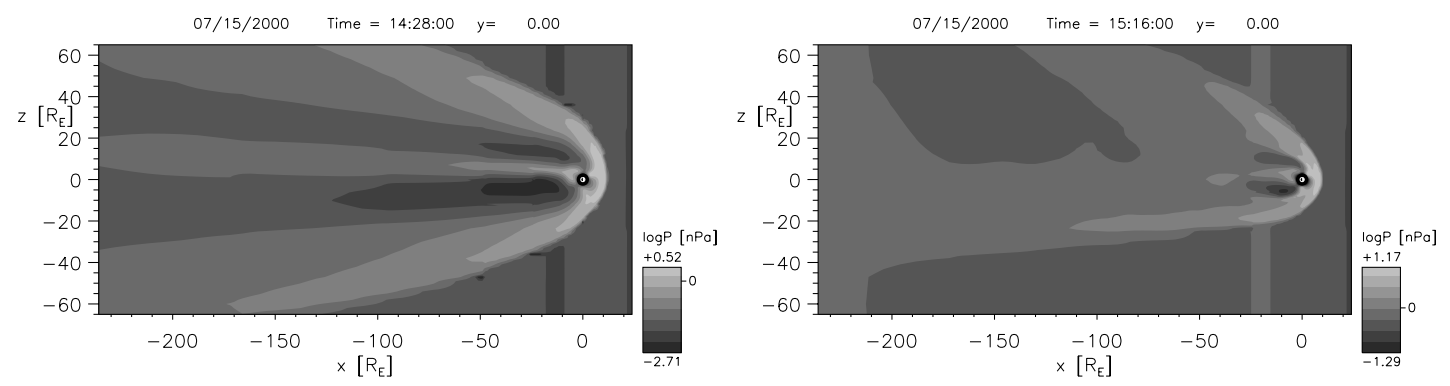

Figure 2. Plasma pressure distribution at 14:28 UT and 15:16 UT. The plasma pressure in the noon-midnight plane at $Y=0$ is shown for the beginning and end of the time period. Note the different scale ranges in the two panels, translating to $[0.00195 \mathrm{nPa}, 3.3 \mathrm{nPa}]$ and $[0.039 \mathrm{nPa}, 14.8 \mathrm{nPa}]$, respectively. 

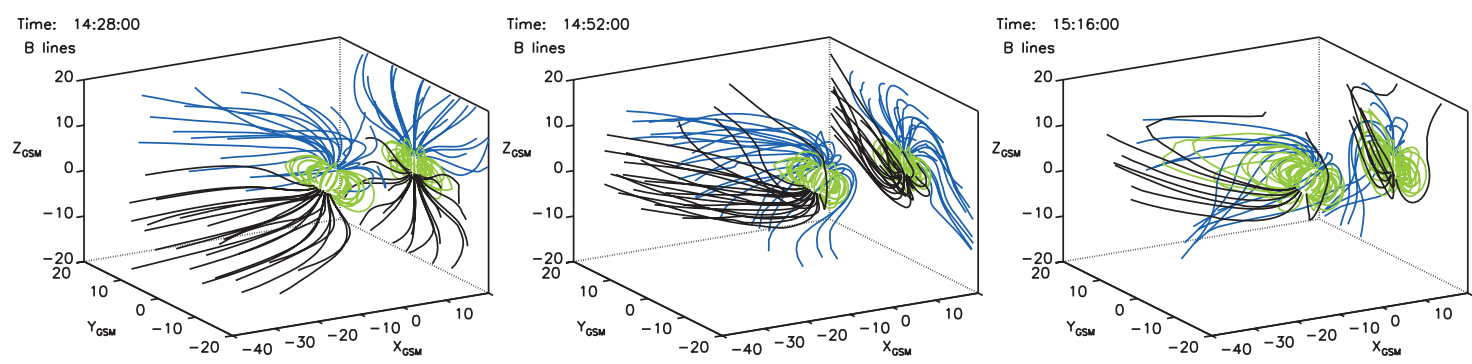

Figure 3. Magnetic field lines at 14:28 UT, 14:48 UT, and 15:16 UT. Closed field lines are light-shaded, open field lines anchored in the northern hemisphere dark, and those anchored in the south are black. The amount of open flux decreases from the initial quiet time (left panel) during this time period (middle, right panels).

Later, as $B_{z}$ remains strongly northward, the inner magnetosphere with closed field lines (light shade) grows and the amount of open flux (dark shaded, black field lines) shrinks further. The IMF field line sections attached to the open field lines shift toward dusk for the northern (dark) and towards dawn for the southern (black) field lines as $B_{y}$ reverses before the end of the time interval.

[9] Figure 4 shows the distribution of the conjugate MLT position $\left(\mathrm{MLT}_{\mathrm{C}}\right)$ of closed field lines starting from the northern hemisphere. Foot points of the field lines in the northern hemisphere are shown in $X_{\mathrm{SM}}$ (vertical axis) and $Y_{\mathrm{SM}}$ (horizontal). Note that $Y$ increases from dawn on the right to dusk on the left. Noon is at the top of each panel. The lines are constant local time in the southern hemisphere $\left(\mathrm{MLT}_{\mathrm{C}}\right)$ mapped on the northern hemisphere. The empty area near the pole (origin) is the polar cap with open field lines. Outside of the dashed circle (located near $60^{\circ}$ magnetic latitude), the lines continue with undistorted mapping $\left(\mathrm{MLT}=\mathrm{MLT}_{\mathrm{C}}\right.$, hours identified by the numbers). The quiettime condition in the left panel (14:28 UT) is characterized by a strong concentration of $\mathrm{MLT}_{\mathrm{C}}$ isolines near noon which is caused by the tilt of the north pole of $\sim 30^{\circ}$ toward the Sun. However, dawn-dusk symmetry is almost perfect. Only a dipole without tilt would show perfect symmetry (i.e. radial lines all the way to the polar cap area). During the passage of the CME by 14:52 UT the polar cap area shrinks from the dawnside and the distribution of the $\mathrm{MLT}_{\mathrm{C}}$ isolines becomes distorted (middle panel). The distortion $\left(\mid \mathrm{MLT}_{\mathrm{C}}-\right.$
MLT|) becomes as large as 9 hours (up from $\leq 5$ hours at 14:28 UT) between the northern and southern foot points for field lines starting near noon. Some single field lines even indicate connections to the exact opposite side indicated by the closely spaced lines along the open-closed field line boundary on the morning side showing that late-afternoon $\mathrm{MLT}_{\mathrm{C}}$ values are encountered on those field lines. The last panel shows that the orientation of the distortion has reversed. The area around noon showing afternoon values of MLT $_{C}$ in the southern hemisphere for late morning MLT foot point position in the north vanishes and gives way to MLT $_{C}$ isolines from the morning side (15:16 UT, right panel). Near midnight we now see pre-midnight MLT $_{C}$ values appearing for field lines starting in the early morning sector. This development can be explained by the shifting locations of cusp reconnection sites in the now negative $B_{y}$ occurring between 15:03 UT and 15:16 UT.

\section{Discussion}

[10] We have shown that the solar wind magnetic field during the Bastille Day Event (July, 15, 2000, after 14:00 UT) generates strong changes of the magnetic topology in the near-Earth magnetosphere. The originally well-organized and almost perfectly (dawn-dusk) symmetric magnetic field line configuration changes into a highly complex tangle of field lines connecting the pre-noon sector in the northern hemisphere to the afternoon and post-midnight sectors in the
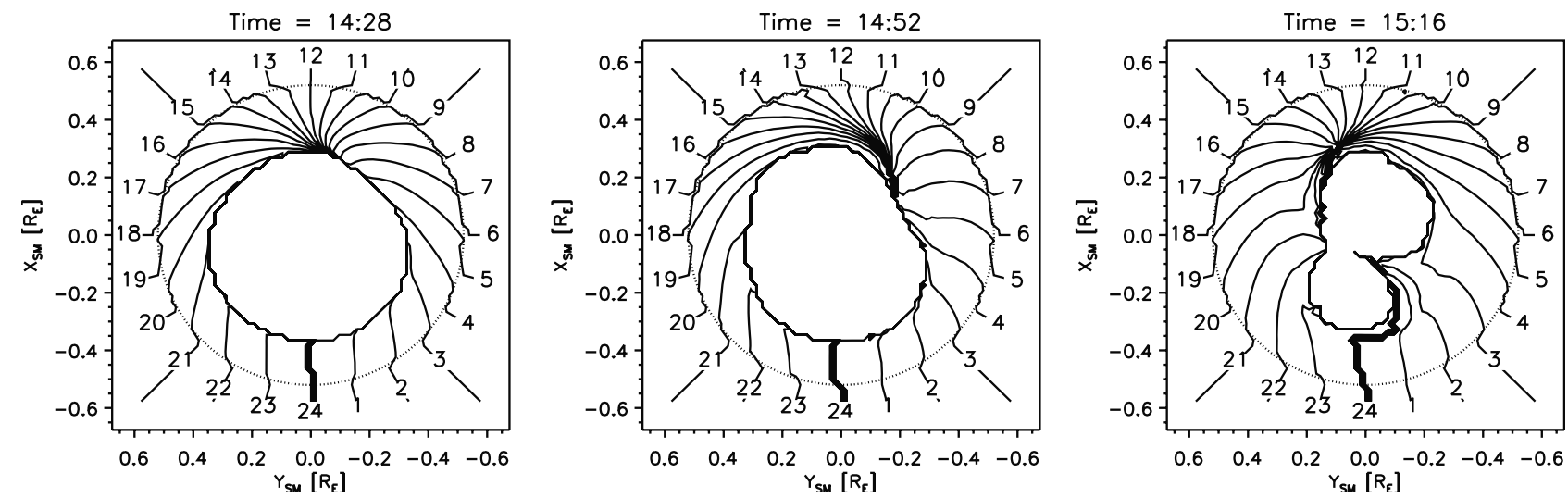

Figure 4. MLT location of conjugate footpoints. For every hour of conjugate magnetic local time (MLT $\mathrm{C}_{\mathrm{C}}$, numbers), the lines show the footpoint location in the northern hemisphere for the same times as in Figure 3. The polar cap (empty region in center) shrinks throughout this time period. 
southern hemisphere. This asymmetry is due to the advection of a strong horizontal component $\left(B_{y}\right)$ of the solar wind magnetic field. Some field lines emanating from near the open-closed field line boundary in the northern hemisphere have conjugate points on the southern hemisphere that are up to 8 hours away in magnetic local time. Changes of IMF $B_{y}$ later during the event reverses the mapping by connecting afternoon sectors in the north to conjugate points in the morning sector in the south. The connection of Earth magnetic field lines to the changing IMF field lines are very similar to those found by [Fedder et al., 1995], for similar IMF conditions (Plate 2), but our study extends their findings by applying the MHD simulation to a real event with changing conditions and by tracing field lines back to conjugate points in the ionosphere.

[11] We can follow the time evolution of the changing field line connections and clearly see the effects of the changing IMF conditions on the magnetosphere. With the July dipole tilt, magnetic reconnection in the northern hemispheric cusp occurs first and with a short delay, reconnection follows in the southern cusp region [Russell, 1972].

[12] After the CME arrives, the open flux in the polar caps begins to reconnect with the now northward IMF in the northern cusp region, connecting them to portions of the IMF field lines south of the reconnection region. The substantial IMF $B_{y}$ component creates the distorted conjugacy maps once these IMF portions reconnect in the southern cusp regions to form new closed field lines as the IMF field is swept downtail. In this process magnetic flux is taken away from the polar cap on opposite sides (morning in the north, afternoon in the south, for example) and the new closed field lines along the polar cap boundary stand out in the MLT maps because of their distinct conjugate footpoints. Once the direction of $B_{y}$ changes, the distribution of the conjugate footpoints of newly closed field lines reverses as well within a time period of a few minutes (now foot points in the afternoon side in the north get connected to morningside footpoints in the south).

[13] The unexpected large distortion of the geomagnetic field found in our simulation constitutes a model prediction. In the future, a combination of modeling and observation should lead to enhanced physical insight. As a next step along these lines, this study will be extended to include polar cap size and cap location comparisons with satellite imaging data to check the validity of the simulation results.

[14] Acknowledgments. This work was supported by the NASA Space Physics Theory Program and was performed within the framework of the Community Coordinated Modeling Center (CCMC) which is supported by NASA, AFMC, AFOSR, AFRL, AFWA, NOAA, and NSF, and ONR. Computations were performed on the IBM SP2 system at the Air Force Weather Agency (AFWA) at Omaha, Nebraska. GEOTAIL solar wind IMF data were obtained through CDAWeb (http://CDAWeb.gsfc.nasa.gov/). $\mathrm{K}$. Ackerson (U. Iowa) provided us with corrected CPI plasma data.

\section{References}

Amm, O., Comment on [Goodman, 1995], Ann. Geophys., 14, 773, 1996. Boris, J. P., A physically motivated solution of the Alfven problem, Tech. Report NRL Memorandum Report 2167, Naval Research Laboratory, Washington, D. C., 1970.

Brittnacher, M., J. Spann, G. Parks, and G. Germany, Auroral observations by the Polar Ultraviolet Imager UVI, Advances in Space Research, 20, 1037-1042, 1997.

Fedder, J. A., J. G. Lyon, Slinker, and C. M. Mobarry, Topological structure of the magnetotail as a function of interplanetary magnetic field direction, J. Geophys. Res., 100, 3613, 1995.

Frank, L. A., K. L. Ackerson, W. R. Paterson, J. A. Lee, M. R. English, and G. L. Pickett, The Comprehensive Plasma Instrumentation (CPI) for the GEOTAIL spacecraft, J. of Geomag. Geoelectr., 46, 23, 1994.

Gombosi, T. I., G. Tóth, D. L. DeZeeuw, K. G. Hansen, K. Kabin, and K. G. Powell, Semi-relativistic magnetophydrodynamics and physics-based convergence acceleration, to appear in J. Comp. Phys., 2002.

Goodman, M. L., A three-dimensional, iterative mapping procedure for the implementation of an ionosphere-magnetosphere anisotropic Ohm's law boundary condition in global magnetohydrodynamic simulations, Ann. Geophys., 13, 843, 1995.

Goodman, M. L., Reply to [Amm, 1996], Ann. Geophys., 14, 775, 1996.

Kokobun, S. T., Y. Yamamoto, M. H. Acuña, K. Hayashi, K. Shiokawa, and H. Kawano, The GEOTAIL Magnetic Field Experiment, J. Geomag. Geoelectr., 46, 7, 1994.

Powell, K. G., P. L. Roe, T. J. Linde, T. I. Gombosi, and D. L. DeZeeuw, A solution-adaptive upwind scheme for ideal magnetohydrodynamics, J. Comp. Phys., 154(2), 284, 1999.

Rasmussen, C. E., and R. W. Schunk, Ionospheric Convection Driven by NBZ Currents, J. Geophys. Res., 92, 4491, 1987.

Russell, C. T., The Configuration of the Magnetosphere, in Critical Problems of Magnetospheric Physics, edited by E. R. Dyer, pp. 1-16, IUCSTP Secretariat, Washington, D. C., 1972.

Sojka, J. J., R. W. Schunk, and M. D. Bowline, Driving a physical ionosphere model with a magnetospheric MHD model, J. Geophys. Res., 102, 22,209, 1997 .

L. Rastätter, M. Hesse, and M. M. Kuznetsova, Code 696, NASA Goddard Space Flight Center, Greenbelt, MD 20771, USA. 